\title{
Chapter 102 \\ Report on Global Environment \\ Competitiveness of New Zealand
}

New Zealand is an island country located in the southwestern Pacific Ocean. New Zealand is situated some $1,500 \mathrm{~km}$ east of Australia across the Tasman Sea and the coastline is $15,134 \mathrm{~km}$. It covers 263.3 thousand of square kilometers and has a population of 4.405 million. Its GDP reaches $\$ 159.71$ billion in 2011 . Through the index system

\begin{tabular}{|l|}
\hline Score: \\
57.67 \\
\hline Rank: \\
4 \\
\hline
\end{tabular}
and evaluation model, the environment competitiveness index of New Zealand ranks at 4 in 133 countries.

III Resource Environment Competitiveness

\# Ecological Environment Competitiveness Environment Carrying Competitiveness

Environment Management Competitiveness Environment Harmony Competitiveness
Fig. 102.1 Contribution of sub-index of GEC

$23 \%$

$24 \%$
$19 \%$

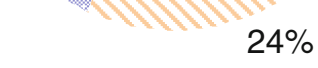




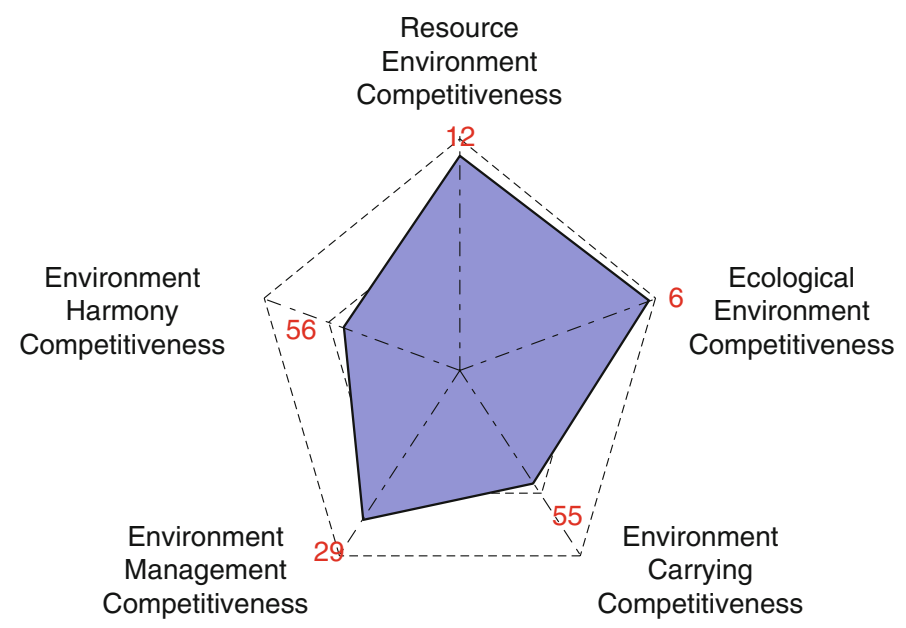

Fig. 102.2 Rank of sub-index of GEC

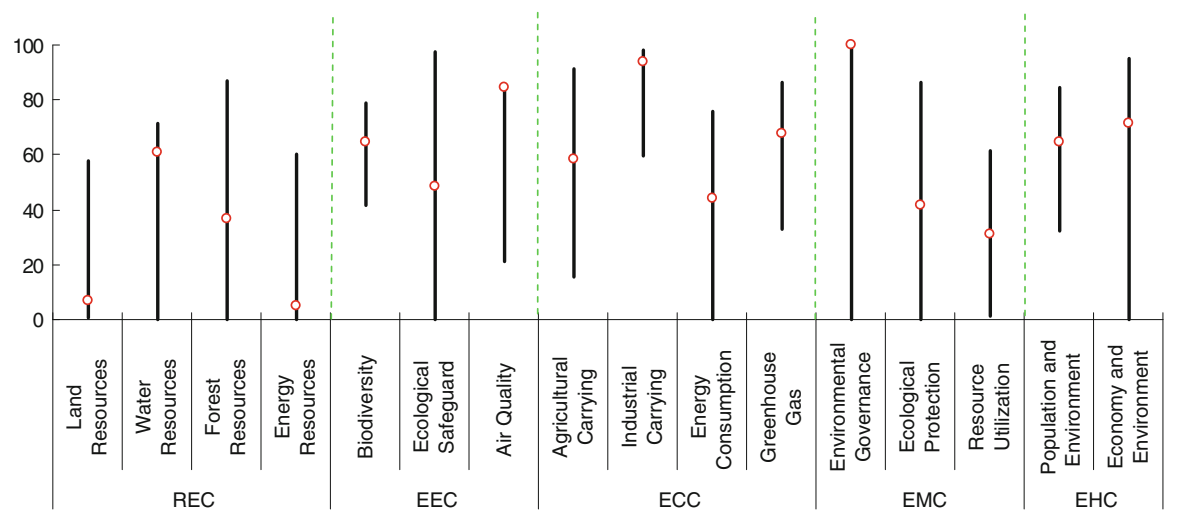

Fig. 102.3 Score and rank of the pillars of GEC

Table 102.1 Score and rank of all indicators of GEC

\begin{tabular}{|c|c|c|c|c|c|}
\hline Indicators & Score & Rank & Indicators & Score & Rank \\
\hline 1 Resource Environment & 28.57 & 12 & Groundwater & N/A & N/A \\
\hline Competitiveness & & & \multirow{2}{*}{$\begin{array}{l}\text { Total internal renewable } \\
\text { water resources }\end{array}$} & \multirow[t]{2}{*}{62.63} & \multirow[t]{2}{*}{12} \\
\hline 1.1 Land Resources & 6.66 & 110 & & & \\
\hline Land area per capita & 10.75 & 24 & 1.3 Forest Resources & 36.88 & 35 \\
\hline $\begin{array}{l}\text { Percentage of arable land to } \\
\text { total land area }\end{array}$ & 2.87 & 119 & $\begin{array}{l}\text { Growing stock in forest and } \\
\text { other wooded land }\end{array}$ & 60.87 & 14 \\
\hline Arable land per capita & 5.00 & 94 & \multirow{2}{*}{$\begin{array}{l}\text { Proportion of land area } \\
\text { covered by forest }\end{array}$} & \multirow[t]{2}{*}{36.74} & \multirow[t]{2}{*}{56} \\
\hline 1.2 Water Resources & 61.17 & 8 & & & \\
\hline Surface water & N/A & N/A & Forest area per capita & 13.08 & 18 \\
\hline Annual precipitation & 59.71 & 21 & 1.4 Energy Resources & 5.05 & 70 \\
\hline
\end{tabular}


Table 102.1 (continued)

\begin{tabular}{|c|c|c|c|c|c|}
\hline Indicators & Score & Rank & Indicators & Score & Rank \\
\hline Fossil energy & 1.79 & 28 & \multirow{2}{*}{$\begin{array}{l}\text { Energy consumption per } \\
\text { unit of land area }\end{array}$} & \multirow[t]{2}{*}{99.86} & \multirow[t]{2}{*}{49} \\
\hline Energy production & 3.90 & 26 & & & \\
\hline $\begin{array}{l}\text { Proportion of combustible } \\
\text { renewable and waste to }\end{array}$ & 6.93 & 70 & $\begin{array}{l}\text { Ratio of clean energy } \\
\text { consumption }\end{array}$ & 50.80 & 8 \\
\hline total energy consumption & & & Elasticity of energy & 13.91 & 67 \\
\hline $\begin{array}{l}\text { Net energy imports of the } \\
\text { energy consumption }\end{array}$ & 11.74 & 47 & $\begin{array}{l}\text { Elasticity of electric power } \\
\text { consumption }\end{array}$ & 11.06 & \multirow[t]{2}{*}{48} \\
\hline $\begin{array}{l}2 \text { Ecological Environment } \\
\text { Competitiveness }\end{array}$ & 67.58 & 6 & $\begin{array}{l}\text { consumption } \\
\text { 3.4 Greenhouse Gas }\end{array}$ & 67.83 & \\
\hline $\begin{array}{l}\text { 2.1 Biodiversity } \\
\text { Threatened fish species }\end{array}$ & $\begin{array}{l}64.71 \\
89.15\end{array}$ & $\begin{array}{r}6 \\
62\end{array}$ & $\begin{array}{l}\text { Growth rate of } \mathrm{CO}_{2} \\
\text { emissions }\end{array}$ & 62.70 & 29 \\
\hline Threatened mammal species & 95.11 & 49 & $\begin{array}{l}\text { Growth rate of Methane } \\
\text { emissions }\end{array}$ & 58.22 & 70 \\
\hline $\begin{array}{l}\text { Threatened plant species } \\
\text { GEF benefits index for } \\
\text { biodiversity }\end{array}$ & $\begin{array}{l}98.89 \\
20.20\end{array}$ & $\begin{array}{l}78 \\
18\end{array}$ & $\begin{array}{l}\text { emissions } \\
\mathrm{CO}_{2} \text { emissions per unit of } \\
\text { land area }\end{array}$ & 99.87 & 56 \\
\hline 2.2 Ecological Safeguard & $\begin{array}{l}48.24 \\
70.92\end{array}$ & 17 & $\begin{array}{l}\mathrm{CO}_{2} \text { emissions per unit } \\
\text { of energy consumption }\end{array}$ & 55.69 & 40 \\
\hline Marine protected areas & 14.21 & 21 & \multirow{2}{*}{$\begin{array}{l}4 \text { Environment Management } \\
\text { Competitiveness }\end{array}$} & \multirow[t]{2}{*}{56.11} & \multirow[t]{2}{*}{29} \\
\hline 2.3 Air Quality & 84.25 & 2 & & & \\
\hline Inhalable particles (PM10) & 91.97 & 7 & \multirow{2}{*}{$\begin{array}{l}\text { 4.1 Environmental } \\
\text { Governance } \\
\text { Agricultural chemicals } \\
\text { regulation }\end{array}$} & 100.00 & 1 \\
\hline $\begin{array}{l}\text { Particulate matter (PM2.5) } \\
\text { Index of indoor air pollution }\end{array}$ & $\begin{array}{r}99.03 \\
100.00\end{array}$ & $\begin{array}{l}8 \\
1\end{array}$ & & 100.00 & 1 \\
\hline Nitrogen oxides emission & 66.25 & 91 & \multirow{2}{*}{$\begin{array}{l}\text { Percentage of the rural } \\
\text { population with access } \\
\text { to an improved water } \\
\text { source }\end{array}$} & \multirow[t]{2}{*}{100.00} & \multirow[t]{2}{*}{1} \\
\hline $\begin{array}{l}\text { Sulfur dioxide emission } \\
3 \text { Environment Carrying } \\
\text { Competitiveness }\end{array}$ & $\begin{array}{l}40.73 \\
67.98\end{array}$ & $\begin{array}{l}59 \\
55\end{array}$ & & & \\
\hline 3.1 Agricultural Carrying & 58.34 & 123 & \multirow{3}{*}{$\begin{array}{l}\text { Percentage of the urban } \\
\text { population with access } \\
\text { to an improved water } \\
\text { source }\end{array}$} & \multirow{3}{*}{100.00} & \multirow[t]{3}{*}{1} \\
\hline $\begin{array}{l}\text { Cereal yield per unit } \\
\text { of arable land }\end{array}$ & 79.19 & 5 & & & \\
\hline $\begin{array}{l}\text { Fertilizer consumption per } \\
\text { unit of arable land }\end{array}$ & 0.35 & 129 & & & \\
\hline $\begin{array}{l}\text { Annual freshwater } \\
\text { withdrawals for }\end{array}$ & 88.54 & 114 & $\begin{array}{l}\text { Area of plantation and } \\
\quad \text { afforestation }\end{array}$ & 2.35 & 24 \\
\hline agriculture per unit & & & Biome protect & 83.90 & 39 \\
\hline $\begin{array}{l}\text { of arable land } \\
\text { 3.2 Industrial Carrying }\end{array}$ & 93.76 & 34 & $\begin{array}{l}\text { Overfishing of fishing } \\
\text { resources }\end{array}$ & 52.50 & 50 \\
\hline $\begin{array}{l}\text { Net exports of goods as a } \\
\text { percentage of GDP }\end{array}$ & 85.39 & 43 & 4.3 Resource Utilization & 31.22 & 89 \\
\hline Electric power consumption & 90.38 & 80 & $\begin{array}{l}\text { Utilization rate of water } \\
\text { resources }\end{array}$ & 0.05 & 107 \\
\hline $\begin{array}{l}\text { per unit of value added } \\
\text { of industry }\end{array}$ & & & $\begin{array}{l}\text { Percentage of total internal } \\
\text { renewable water }\end{array}$ & N/A & N/A \\
\hline $\begin{array}{l}\mathrm{SO}_{2} \text { emissions per unit of } \\
\text { value added of industry }\end{array}$ & 99.92 & 33 & $\begin{array}{l}\text { resources to total water } \\
\text { resources }\end{array}$ & & \\
\hline $\begin{array}{l}\text { Annual freshwater } \\
\text { withdrawals for industry }\end{array}$ & 99.33 & 27 & $\begin{array}{l}\text { Percentage of agricultural } \\
\text { land to total land area }\end{array}$ & 51.58 & 66 \\
\hline $\begin{array}{l}\text { per value added of } \\
\text { industry }\end{array}$ & & & $\begin{array}{l}\text { Percentage of fossil fuel } \\
\text { energy consumption to }\end{array}$ & 42.03 & 38 \\
\hline 3.3 Energy Consumption & 43.91 & 15 & total energy consumption & & \\
\hline
\end{tabular}


Table 102.1 (continued)

\begin{tabular}{lcclcc}
\hline Indicators & Score & Rank & Indicators & Score & Rank \\
\hline $\begin{array}{l}5 \text { Environment Harmony } \\
\text { Competitiveness }\end{array}$ & 68.13 & 56 & $\begin{array}{l}\mathrm{CO}_{2} \text { emissions per capita } \\
\text { Energy consumption } \\
\text { per capita }\end{array}$ & 81.01 & 100 \\
$\begin{array}{c}5.1 \text { Population and } \\
\quad \text { Environment }\end{array}$ & 64.85 & 99 & $\begin{array}{l}68.20 \\
\text { 5.2 Economy and } \\
\text { Environment }\end{array}$ & 71.41 & 22 \\
$\begin{array}{c}\text { Percentage of population } \\
\text { with access to Improved } \\
\text { sanitation facilities }\end{array}$ & N/A & N/A & $\begin{array}{c}\text { Land resource utilization } \\
\text { efficiency }\end{array}$ & 0.18 & 51 \\
$\begin{array}{c}\text { Motor vehicles per 1,000 } \\
\text { people }\end{array}$ & 11.60 & 130 & $\begin{array}{c}\text { Sulfur dioxide emissions } \\
\text { per unit of GDP }\end{array}$ & 98.06 & 27 \\
$\begin{array}{c}\text { Renewable internal } \\
\text { freshwater resources per } \\
\text { capita }\end{array}$ & 89.82 & 4 & $\begin{array}{c}\text { Carbon dioxide emissions } \\
\text { per unit of GDP }\end{array}$ & 93.91 & 28 \\
$\mathrm{SO}_{2}$ emissions per capita & 91.37 & 90 & $\begin{array}{c}\text { Energy consumption per } \\
\text { unit of GDP }\end{array}$ & 93.48 & 26 \\
\hline
\end{tabular}

Table 102.2 Rank distribution of the individual indicators of GEC

\begin{tabular}{llcllll}
\hline & $\begin{array}{l}\text { Number } \\
\text { of the } \\
\text { individual }\end{array}$ & Rank & Rank & Rank & Rank & Rank \\
indicators & $1-10$ & $11-30$ & $31-60$ & $61-100$ & $101-133$ \\
\hline Sub-index & 14 & 1 & 5 & 2 & 2 & 2 \\
\hline $\begin{array}{l}\text { Resource Environment } \\
\quad \text { Competitiveness }\end{array}$ & 11 & 5 & 2 & 1 & 3 & 0 \\
$\begin{array}{c}\text { Ecological Environment } \\
\quad \text { Competitiveness }\end{array}$ & 15 & 2 & 3 & 5 & 3 & 2 \\
$\begin{array}{c}\text { Environment Carrying } \\
\quad \text { Competitiveness }\end{array}$ & 10 & 3 & 1 & 2 & 2 & 1 \\
$\begin{array}{c}\text { Environment Management } \\
\text { Competitiveness }\end{array}$ & 10 & 1 & 3 & 1 & 3 & 1 \\
$\begin{array}{c}\text { Environment Harmony } \\
\text { Competitiveness }\end{array}$ & 60 & 12 & 14 & 11 & 13 & 6 \\
\hline Total & & & & & & \\
\hline
\end{tabular}

Open Access This chapter is distributed under the terms of the Creative Commons Attribution Noncommercial License, which permits any noncommercial use, distribution, and reproduction in any medium, provided the original author(s) and source are credited. 